\title{
A Study of Distribution of Pulmonary Mycobacterium avium Complex (MAC) Disease Cavities and their Course under Chemotherapy
}

\section{Kurashima $\mathrm{A}^{1^{*}}$ and Horibe $\mathbf{M}^{2}$}

${ }^{1}$ Fukujuji Hospital, Japan Anti-Tuberculosis Association, Department of Respiratory Medicine, Japan

${ }^{2}$ Department of Radiology, National Hospital Organization Tokyo Hospital, Japan

\section{Case Report}

Recently, Chu et al. compared the chest imaging features of 210 cases of sputum acid fast bacillus (AFB)-smear positive pulmonary tuberculosis (PTB) to those of 124 cases of non-tuberculous mycobacteria lung disease (NTM-LD), and reported that patients with NTM-LD had bronchiectasis in the middle lobe lingual segment more frequently than those with PTB [1]. While the difference between bacterial species causing NTM-LD were not under consideration in the Chu et al.s study, we previously conducted a study that identified Mycobacterium avium complex (MAC) as a cause of NTM-LD and performed imaging of the cavities. We reported our results in 2012 [2]. We focused on MAC because the occurrence of pulmonary MAC disease (MAC-LD) is the most frequent in many countries, and the reason for performing imaging of the cavities was that the presence of cavities had a major clinical effect on the course of the disease and was the most important factor in choosing surgical treatment.

We conducted a retrospective study of 47 patients with MAC-LD selected according to the diagnostic criteria of the ATS/IDSA2007 guidelines. Only patients who had been treated with combination chemotherapy consisting of clarithromycin (CAM), rifampicin (RFP), and ethambutol (EB) were enrolled. We also included 80 patients with bacteria-positive PTB at the initial examination. All cases had cavities that could be seen on CT images. The segments were named conforming to the Boyden classification; however, some were changed as follows.

\section{Right lung}

Right upper lobe

- Apical segment (S1)

- Posterior segment (S2)

- Anterior segment (S3)

Right middle lobe

- Lateral segment (S4)

- Medial segment (S5)

Right lower lobe

- Superior segment (S6)

- Medial segment (S7)

- Anterior segment (S8)

- Lateral segment (S9)

- Posterior segment (S10)

\section{Left lung}

\section{Left upper lobe}

Apicoposterior segment (S1/2).
The left apicoposterior segment was divided into apical and posterior subsegments, so that the upper parts of the left and right sides correspond to each other. lung

S1 = Apical subsegment of the apicoposterior segment of the left

S2 $=$ Posterior subsegment of the apicoposterior segment of the left lung

Anterior segment (S3)

Superior lingular segment (S4)

Inferior lingular segment (S5)

Left lower lobe

- Superior segment (S6)

- Anteromedial segment (S8)

- Lateral segment (S9)

- Posterior segment (S10)

First, as shown in Figure 1, the cavity distribution at the initial examination was as follows: S1, 10.2\%; S2, 34.7\%; S3, 20.4\%; S4, 4.1\%; S5, 2\%; S6, 6.1\%; S7, 0\%; S8, 4.1\%; S9, 10.2\%; and S10, 8.2\% in the cases with MAC-LD and S1, 41.6\%; S2, 13.3\%; S3, 19.1\%; S4, 3.5\%; S5, 2.3\%; S6, 12.7\%; S7, 3.5\%; S8, 3.5\%; S9, 1.7\%; and S10, $1.7 \%$ in the cases with bacteria-positive PTB. In the analysis of variance of the cavity distribution in both groups, the $\mathrm{F}$ value was 8.95 and the $\mathrm{p}$ value was 0.0031 , a significant difference. A statistical analysis was performed using JMP version 9.2 software. In other words, the cavity distribution of cases with bacteria-positive PTB was often observed in S1 and S6 while it was often observed in S2, S9, and S10 in cases with MAC-LD. Second, we measured the change in cavity size before and after chemotherapy in the 56 cavities in 47 cases with MAC-LD, and the results revealed a reduction in the average outer diameter of 3.1 $\mathrm{mm}(\mathrm{p}=0.032)$. The size of cavities was measured using the method shown in Figure 2.

In addition, the cases were divided into upper and lower lobe

${ }^{*}$ Corresponding author: Dr. Atsuyuki Kurashima, Fukujuji Hospital, Japan AntiTuberculosis Association, Department of Respiratory Medicine, Japan, Tel: +81 42491 4111, Fax: +81 42492 465; E-mail: kurashimaa@fukujuji.org

Received January 27, 2016; Accepted March 26, 2016; Published March 29, 2016

Citation: Kurashima A, Horibe M (2016) A Study of Distribution of Pulmonary Mycobacterium avium Complex (MAC) Disease Cavities and their Course under Chemotherapy . J Antimicro 2: 111. doi: 10.4172/2472-1212.1000111

Copyright: (C) 2016 Kurashima A, et al. This is an open-access article distributed under the terms of the Creative Commons Attribution License, which permits unrestricted use, distribution, and reproduction in any medium, provided the original author and source are credited. 
Citation: Kurashima A, Horibe M (2016) A Study of Distribution of Pulmonary Mycobacterium avium Complex (MAC) Disease Cavities and their Course under Chemotherapy . J Antimicro 2: 111. doi: 10.4172/2472-1212.1000111

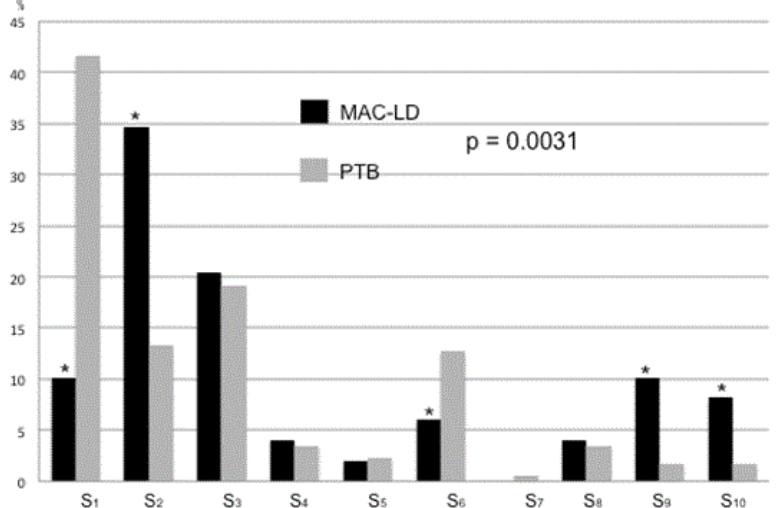

Figure 1: Frequency of initial cavities location according to pulmonary segments. * indicates the segment with asignificant difference between MAC-LD and PTB on $a x^{2}$ test.

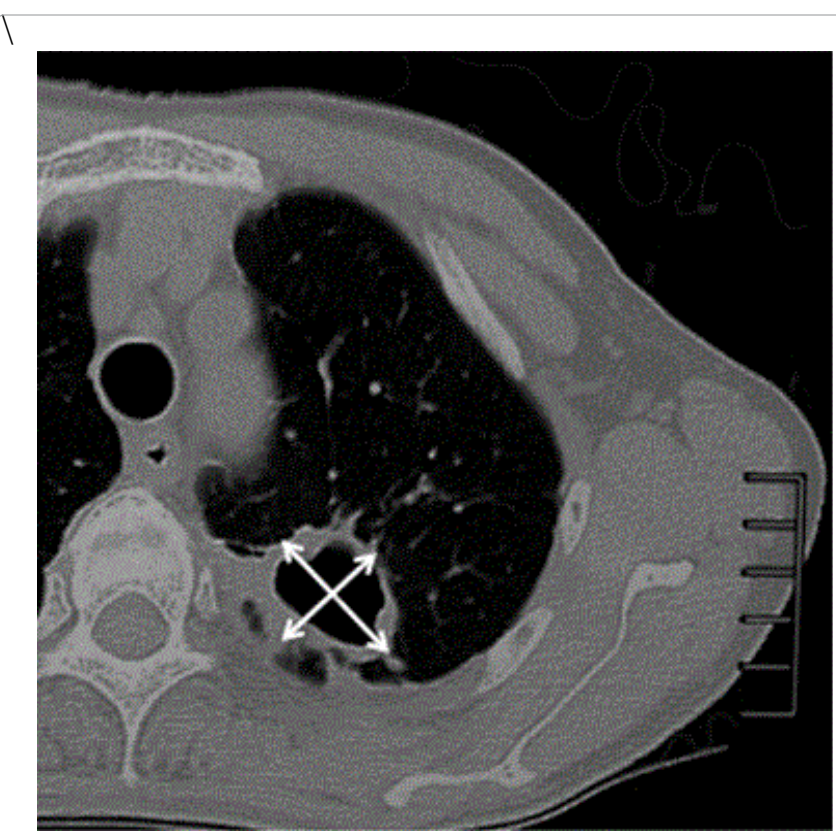

Figure 2: The cavity diameters are defined by the average value of the maximum and minimum external diameter on the CT image. Cavity measurements were performed independently by each of the two authors, and if the measurements were different, we used the average of the two measurements.

groups (Figure 3). The average outer diameters of cavities at the start of chemotherapy were $19.3 \mathrm{~mm}$ and $18.2 \mathrm{~mm}$ in the upper and lower lobe group, respectively, without a significant difference between groups. On the other hand, the average outer diameters of cavities after chemotherapy (the average length was 22 months) was reduced by $0.93 \mathrm{~mm}$ in the upper lobe group while it was reduced by $6.72 \mathrm{~mm}$ in the lower lobe group, a significant difference $(p=0.047)$. In cases with reduction of cavity size, the average number of elapsed days was 888.8 and 464.1 days in the upper and lower lobe groups, respectively; thus, the duration was significantly shorter in the lower lobe group $(\mathrm{p}=0.0215)$.

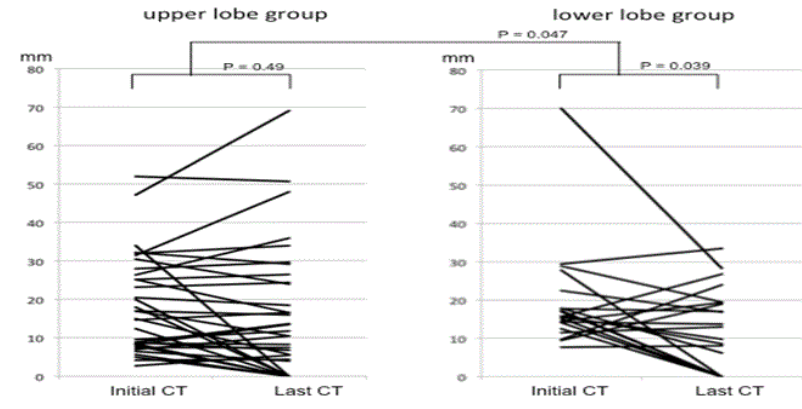

Figure 3: If the cavity changed, then we investigated that change, considering cavities of the upper right lobe as belonging to the "upper lobe group", those of the middle and lower lobe as belonging to the "lower lobe group", and for the left lung, those of the lingula and lower lobe as belonging to the "lower lobe group", and others as belonging to the "upper lobe group". This figure shows the course of cavity diameter change in the upper lobe group and in the lower lobe group.

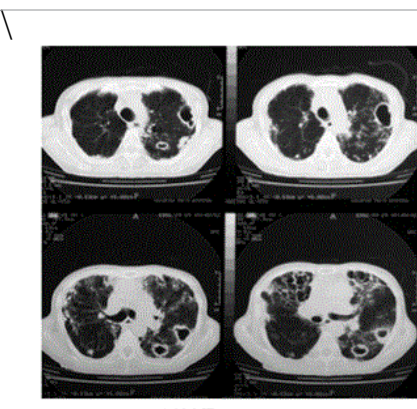

Initial C

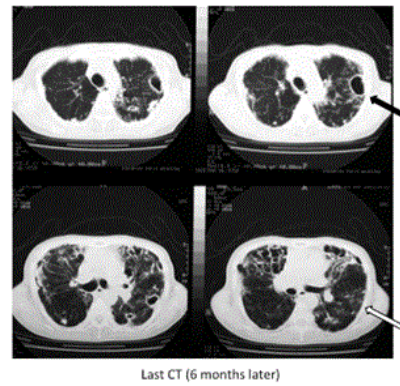

Last CT $(6$ months late)
Figure 4: The difference of cavity reduction at the location belonging to the upper lobe and the lower lobe in the same case. The filled arrow shows the upper lobe cavity which size little changed. The open arrow indicates the lower lobe cavities which were disappeared.

Figure 4 shows the one of CT images, which has plural cavities at the same time. This is the first report of a difference in the cavity distribution between cases with PTB and MAC-LD, and the first observation that a greater reduction in cavity size after chemotherapy was apparent in the lower lobe in cases with MAC-LD. The difference in cavity distribution between cases with PTB and MAC-LD may be due to the difference in the optimal $\mathrm{pH}$ range of MAC bacteria for growth, and the difference between the upper and lower lobe in the reduction of cavity size after chemotherapy in cases with MAC-LD may be caused by differences in the distribution of pulmonary blood flow.

\section{References}

1. Chu HQ, Li B, Zhao L, Huang DD, Zhang ZM, et al. (2015) Chest imaging comparison between non-tuberculous and tuberculosis mycobacteria in sputum acid fast bacilli smear-positive patients. Eur Rev Med Pharmacol Sci 19: 2429-2439.

2. Kurashima A, Horibe M (2012) Distribution of pulmonary Mycobacterium avium complex (MAC) disease cavities and their course under chemotherapy. Kekkaku 87: 397-402. 